\title{
Early Strokes Detection of Patient and Health Monitoring System Based On Data Analytics Using K- Means Algorithm
}

\author{
Menaka. $\mathrm{S}^{1}$, Bharathiraja. $\mathrm{N}^{2}$, Badi Alekhya ${ }^{3}$, Sasikumar. $\mathrm{R}^{4}$ \\ \{menakas311@gmail.com ${ }^{1}$, rajamesoft@gmail.com ${ }^{2}$, alekhyareddy1@gmail.com ${ }^{3}$,rsn.cse@rmd.ac.in ${ }^{4}$ \} \\ ${ }^{1}$ Assistant Professor, Department of Computer Science and Engineering, SRM Institute of Science \\ and Technology, Ramapuram Campus, Chennai, India., ${ }^{2}$ Assistant Professor, Department of Computer \\ Science and Engineering, SRM Institute of Science and Technology, Ramapuram Campus, Chennai, \\ India., ${ }^{3}$ Research Scholar, Department of ICE, R.M.D Engineering College, Kavaraipettai, Chennai, \\ India., ${ }^{4}$ Professor, Department of Computer Science and Engineering, R.M.D Engineering College, \\ Kavaraipettai, Chennai, India.
}

\begin{abstract}
The processing chain of scientific information in particular consists of information series, information storage, data sharing, and records evaluation. In the prevailing device, there is lots of improvisation wished for our health care device. The existing technique our affected person monitoring is a guide and time-consuming process. To conquer the task the proposed work proposed actual data series from sensors, IoT-primarily based totally sharing, and information analytics. This proposed device gives the gain for the respective medical doctor to display the affected person's health $24 * 7$ regardless of geographical location. Example: The medical doctor can display the affected person's health even after the affected person receives discharged. This proposed work implies a health sensor named heartbeat sensor to display affected person fitness. The affected person information is monitoring through the sensorsand transmitted to the Arduino. The actual-time statistics from the COM port have acquired the usage of Net beans and stored in an SQL database. The actual-time information may be monitored with the aid of using each affected person and medical doctor. The real-time statistics are processed from Net beans as datasheet to R programming for statistical evaluation. For Clustering, we use the K-Means algorithm and for Classification, we use the Support Vector Machine. Also relying on the affected person's health situations emergency pills or injections are counseled routinely with the aid of using our device. Also, the affected person statistics have encrypted the usage of an ABE (Attribute-based Encryption) set of rules and saved in the public cloud particularly Dropbox. Thus invoking the statistics evaluation approach facilitates in identifying early stroke in the sufferers and offer medicinal diagnosis immediately.
\end{abstract}

Keywords: Heart-Beat Sensors, Attribute-Based, Encryption, K-Means, Support vector Machine.

\section{Introduction}

The creation of massive information in healthcare has paved the manner for brand spanking new possibilities in the remedy of sufferers, inclusive of precision medicine, drug development, and superior most cancers therapy. It additionally results in technological 
improvements throughout the quarter, inclusive of supplemented stronger health tracking, and the progressed renovation of electronic health records (EHRs). When imposing new analytical software programs and tools, workflow and standard-of-care also can be progressed. By contemplating workflow traits of the latest system as compared to the antique system, it permits facts to be collated to justify a return on investment. The analytical software program also can be used to reveal information and installation signals for different scientific processes inclusive of near radiation, the quantity of time affected person attending clinics, and additionally the procedural instances in labs. The world has visible a big inflow of robots and generation in the industry. However, they aren't taking on the healthcare quarter simply yet. Phrases inclusive of synthetic intelligence and gadget studying are starting to take maintain in the quarter, however, a human touch is nevertheless favored when it involves remedy. The emerging generation is converting the structure of healthcare support. But to make it useful human tracking is nevertheless a required factor, mainly concerning affected person interaction. When receiving a diagnosis, an affected person will, of course, locate it extra comforting to get hold of that facts from a human.. Technology is now making it easier for human beings to navigate existence and manipulate on their own. However, in healthcare, it is still in different from emotion, as one-to-one engagement is nevertheless the maximum green manner to treat sufferers.

The implementation of massive information increases the possibility for massive capacity advantages for healthcare. From use in normal obligations inclusive of information access to superior strategies like tracking sufferers, information is, in reality, shaping the manner of the way we stay and think. Big data has given health care a massive possibility to assess the excellent of remedy. By the use of best algorithms reviews may be created based on standards inclusive of health practitioner training, e-book history, modern institute, and affected person outcomes. It is likewise lifting the geographical barriers confronted via way of means of healthcare, permitting rural international locations to get hold of primary remedy and the get right of entry to a noticeably certified physician. However, ultimately, the goal is to offer the affected person's health information to make the most important choices concerning their remedy and care to make shipping loads extra efficient. Matching sufferers to the maximum experienced (and suitable) physicians for his or her unique wishes from the start.

\section{Related Work}

The current work focuses mostly on fine-grained privacy-preserving access and analysis of static medical text, which can hardly afford the rapid fluctuation of health conditions and analysis of medical images. No healthcare monitoring framework based on IOT was being introduced. Even with wearable devices, the patient is only able to access his health records. The current framework does not have a single architecture that gathers and analyses real-time patient health data using Big Data techniques. In the new method, only the patient will know after the stroke, i.e. only the patient will know that he/she is in the stroke by experiencing the outward symptoms. [3] Hybrid clustering technique is used in this existing work. Provide accurate results. Hybrid approach takes more execution time when compared to Remaining clustering techniques.[4] Google Trends, Data visualization, Correlation analysis, Dynamic regression approach. Forecasting patient volume of data in emergency. Need more man power 
to maintain and privacy issues?[5] Integration tools and techniques. It provides different ideas to integrate data.

Especially, unstructured data by using big data tools and it needs experimental results to understand the effectiveness of Data integration tools.[6] The tool used in this paper helpful to classify ECG signals for early diagnosis Detection. ML-Libs and scale Language on Apache Spark. Limited to some classes of classification and datasets.[7] The technique used here explains multi class classification of heart beats from ECG signals.2D-Deep Convolution-al neural network, Short- Term Fourier Transform. The technique used single DB (MIT-BIH) not consider the experimental results of different DB's to compute Accuracy.[10] The techniques used here provide the signal quality importance in ECG signals under unsupervised diagnosis environments. Complete ensemble empirical mode decomposition (CEE MD) algorithm-peak Detection approach. It limits the experimental results to accept the noiseless signals and reject the noisy signals under unsupervised health monitoring. [11]It develops the precise learning algorithms that synthesize dynamic time deformation, C-means clump, and BP algorithmic program.

The 12-lead cardiogram (ECG) signals for cardiopathy identification were used for classification testing. The experimental results from 12-lead electrocardiogram signal across 10 forms of wellness classification verify the effectiveness of the model and therefore the projected algorithmic program. [12]Recently, good attention applications AR being fitted with wearable sensors, that AR principally accustomed monitor and strengthen the human action recognition (HAR) mistreatment supervised and unsupervised learning ways that fail to realize reduced computation time to on-nodule wearable sensors and through the process of knowledge within the network, it fails to cut back the reconstruction error rate with optimized accuracy throughout classification. [13] This instructed, associate degree innovative, unsupervised Deep learning motor-assisted reconstructed software engineer (UDR-RC) that optimize the info throughout pre-processing at on-nodule wearable sensors to induce reduced computation time.[14] Implementation of machine-learning-based patient processing is influenced by heterogeneous patient knowledge and inefficient in analysing feature-learning ways.[15]

This works transform such every which way ordered forward/backward firing sequence of transitions within the network into a set topological order of transition-firing in forward direction solely by exchange backward firing transitions into equivalent forward firing transitions. [16]Automatic detection and classification of noises will play an important role within the development of sturdy unattended graphical record (ECG) analysis systems. This paper proposes a unique unified framework for automatic detection, localization, and classification of single and combined electrocardiogram noises. ways . The projected framework consists of the changed ensemble empirical mode decomposition (CEEMD), the short-run temporal feature extraction, and also the decision-rule-based noise detection and classification.[17] Stroke was diagnosed by a specialist in a very hospital at or on top of the county level supported the self-reported history of stroke and so computed axial tomography or resonance imaging. Nonfatal cerebrovascular accident, cerebrovascular accident, and transient anaemia attack were all enclosed. The case history was outlined as a history of stroke in oldsters, grandparents, aunts or uncles, and 1st cousins. The smoking and uptake habits of those farmers are discovered. 


\section{Proposed Model for Patient and Health Monitoring System}

In this proposed work, the proposed health sensor named heartbeat sensor to track the health of patients The real and complex patient data is transmitted to the system using the Ethernet cable, and the patient information is gathered on or after the sensors and transmitted to the Arduino. The real-time COM port statistics were acquired using the Arduino IDE and stored in the SQL database. Both the patient and the doctor will track the real-time data. This was achieved by exporting an excel file from the Arduino Software to the $\mathrm{R}$ programming studio for data analysis. We use the K-means algorithm for clustering and we use the Help Vector Machine algorithm for classification. Emergency medicines or injections are often proposed based on the patient's health conditions through our system automatically. The affected person, too, statistics have encrypted the usage of an Attribute-primarily based totally Encryption set of rules and saved in the public cloud namely Dropbox.

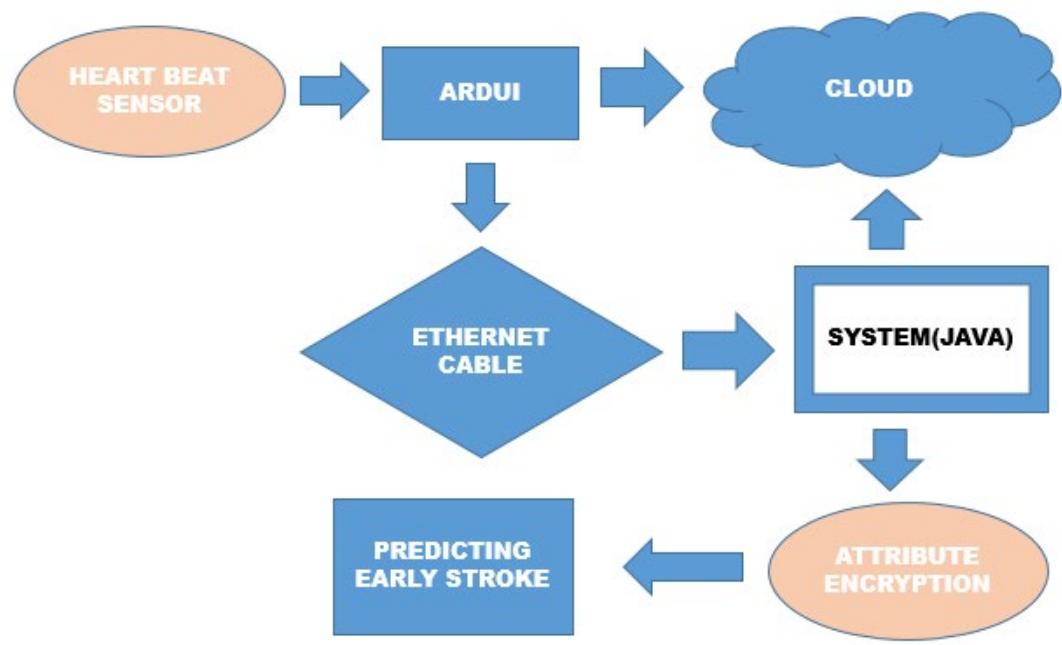

Fig 1: System Design for Patient and Health Monitoring System

\section{Experimental Results}

The Heart Rate sensor video display units the mild degree transmitted via the vascular tissue of the fingertip and the corresponding versions in mild. Intensities that arise as the blood extent extrudes in the tissue. The ease of use makes it feasible to degree everyone's coronary heart price, even in huge classes. 


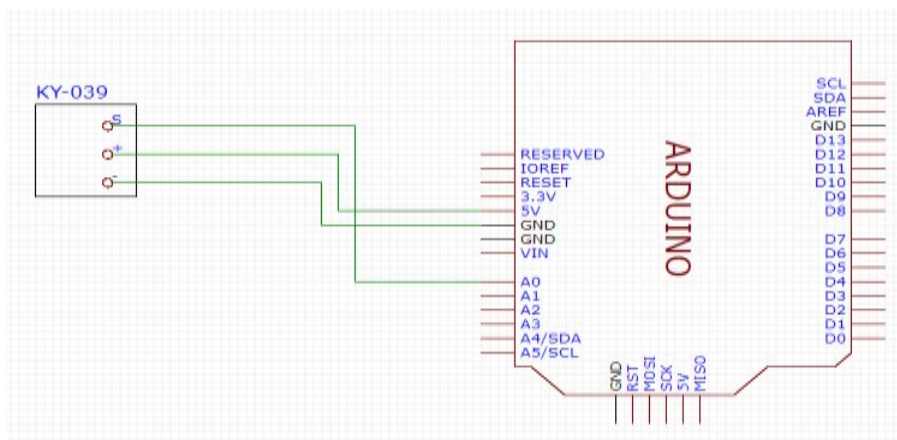

Fig.2 Arduino

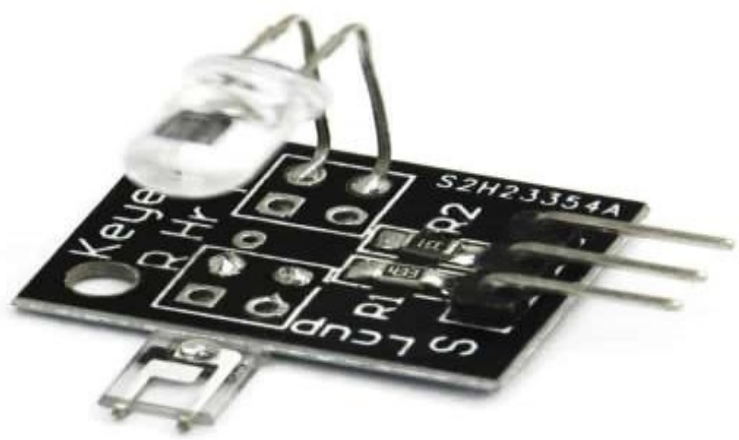

Fig.3 ATmega328

The Heart Rate sensor measures a coronary heart rate between 0 and 200 bpm (beats per minute).The Arduino Uno is a microcontroller sensor module also on ATmega3288 (datasheet). It has 14 virtual I/O pins, including six PWM outputs, six analogue inputs, a 16 MHza USB connection, a power jack, a reset button, and an ICSP header. It must provide full assistance to the microcontroller. To get started, obviously attach to power with an AC-to-DC adapter or a computer with a USB cable. The FTDI USB-to-serial driving force chip is no longer used in the Uno, as it was in previous forums. Instead, the Atmega8U2 is set up to act as a USB-to-serial converter. The name "Uno" is a Italian word "uno," meanings "one." It is named after the upcoming Arduino 1.0.0 Version 1.0 of are the reference versions of Arduino that will be used in the future. The Uno is the most recent in a series of Arduino USB boards and the platform's reference model for a comparison of previous versions, see the Arduino board index.

API (Application Program Interface) by Java developers offers all functionality of serial communications. To render the API portable across platforms, it defines an abstract Serial Port class. This class is then converted into a child class's entity, which includes platform-specific functionality. This class then interacts with a Dynamic Connection Library (DCL) through the Java Native Interface (JNI). Once a Serial Port object has been developed, standard Input Stream and Output Stream objects perform communications through the physical port. Bytes, integers, and arrays of bytes are used to send and receive data in these streams. 


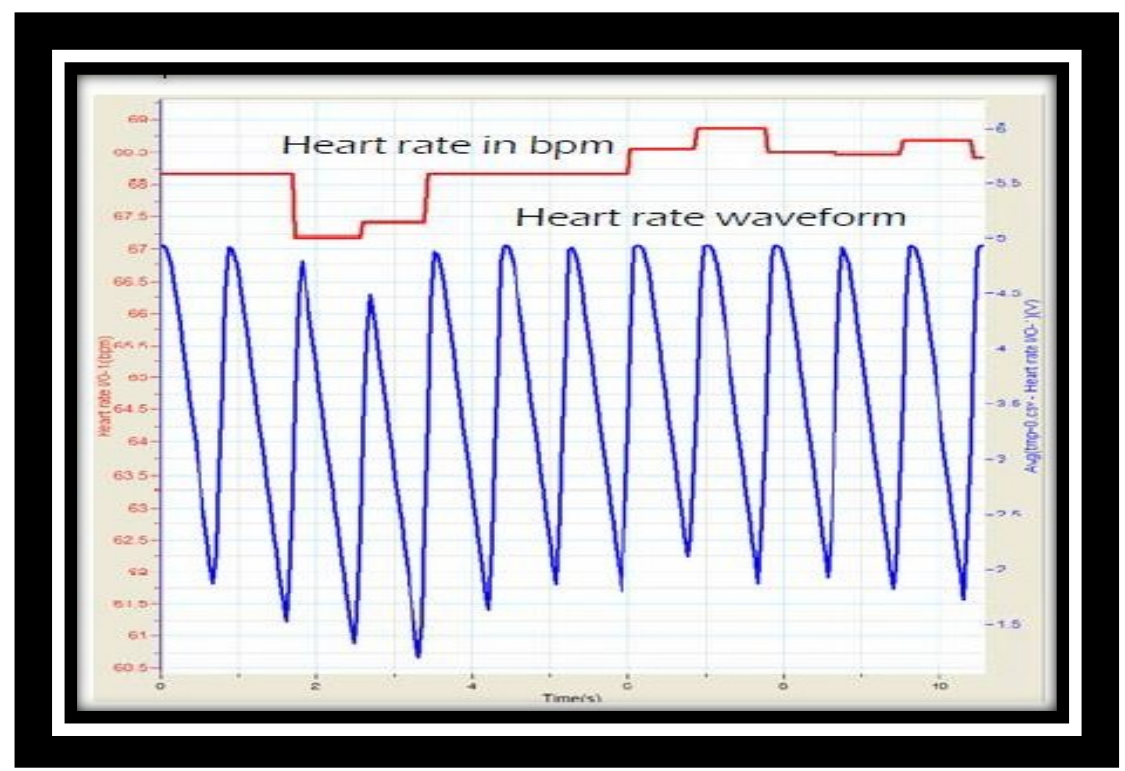

Fig 4: Monitoring both heart rate in bpm and waveform

$\mathrm{ABE}$ (attribute-based encryption) is a modern technique that rethinks the idea of publickey encryption. A message is encrypted using the receiver's public key for a specific receiver in traditional public key cryptography. Identity-based encryption and, particularly, identitybased encryption (IBE).The standard concept of public-key cryptography has modified by allowing the public-key to be any string, such as the recipient's email address. It is possible to encrypt roles and messages in relation to attribute subsets (key-policy ABE - KP-ABE) or policies which defines the identity as a set of attributes $\mathrm{ABE}$ rather than an atomic identity(cipher text- policy ABE - CP-ABE). The important challenge is that an user will only be able to retrieve a cipher text whether they have a key for "corresponding attributes" (see below), which is often provided by a trusted third party. 


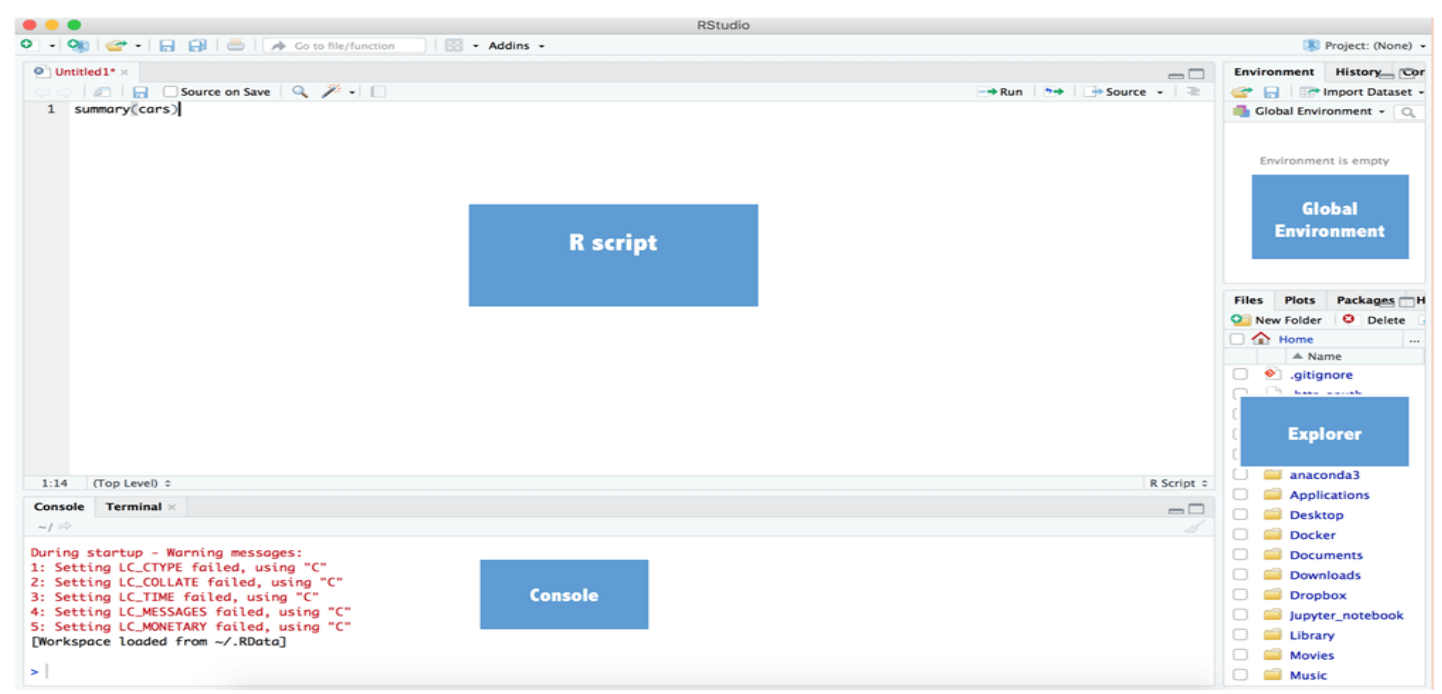

Fig 4: Perform Analytics

Cloud storage is a data processing model where virtual data are stored pools, where computing power covers multiple servers (and sometimes locations), and where a web host normally maintains and operates the physical environment. These cloud storage services are responsible for keeping the data available and accessible, and safeguarding and running the physical environment. Individuals and organizations purchase or lease computing space from vendors to store information from users, companies, or applications.

$\mathrm{R}$ is a scientific method, visualization representation and reporting programming language and software environment. In different areas, such as marketing, informatics and geo-spatial fields, a large amount of large databases has been obtained. Big data mining expertise is becoming a highly demanding field. It far surpassed the capacity of humans to analyse these enormous results, however. Unsupervised Machine Learning or Clustering is one of the most powerful data mining methods for discovering information in large datasets.

\section{Conclusion}

In this proposed work, the idea introduced real time data analytics using sensors and machine learning techniques to predict early stroke and save human lives. Here, he/she will be able to view the affected person listing with precedence device in step with their threat stage and he also can view the affected person's info and former analysis results. Besides, after clicking at the monitoring device button he/she will be able to visualize every affected person's health repute and real-time physiological information. In this work, Heart Rate Waveform also can be related to the Arduino in order to be able to transmitting real-time information to the server. The sensor isn't always introduced to the designed affected person tracking device because of the unavailability of a clinically diagnosed device at this moment. Though a version of the cloud-primarily based totally coronary heart sickness detection utility is represented here, the destiny works may be centered at the improvement of a committed server and database for this kind of affected person tracking utility. If the 
proposed utility may be included and evolved successfully, it'll be to be had with inside the Android play save consequently. Thus, any affected person or medical doctor from any location of the globe may be able to putting in this utility and use this utility for coronary heart sickness prediction as reported. The experimental results provide promising results.

\section{References}

[1] In search of Medical Integrated solutions-A Comprehensive Survey, published by IEEE Access, 2019,vol 7,pg no91265-91290

[2] Big Data Features, Applications and Analytics in cardiology-A Systematic literature Review, published by IEEE Access, 2019,vol 7,pg no143742-143770

[3] Sunil Kumar ,Maninder Singh,” A Novel Clustering technique for Efficient clustering for BigData in Hadoop Ecosystem”, published by BigData Mining and Analytics ,2019, vol 2,pgno 240-247

[4] Andrew Fu Wah Ho, Bryan Zhan Yuan Se ,Jin Ming Koh,'Forecasting Hospital Emergency Department patient volume using Internet search data", published by IEEE Access 2019,vol 7,pgno 93387-93395

[5] HousseinDhayne , Rafiqul Haque,rima Kilany1, And Yehia Taherin, "Search Of Medical Integrated Solutions-a Comprehensive Survey", published by IEEE Access 2019,vol 7,pgno91265-91290

[6] FajrlbrahemAlarsan, and Mamoon Younes, "Analysis and Classification of heart diseases using heart beat features and machine learning algorithms", published by Journal of Bigdata(Springer),2019,pgno1-15

[7] Jingshan Huang , Binqiang Chen , Bin Yao,AndWangpeng He, "ECGArrhythmia Classification Using STFT-Based Spectrogramand Convolutional Neural Network”, published by IEEE Access 2019,vol7,pgno 92871-92880.

[8] Menaka. S, Rohini. S, "Secure the Data by using Image Compression in Multi-Cloud Storage," International Journal of Engineering and Advanced Technology (IJEAT), ISSN: 2249 - 8958, Volume-9, Issue-1S2, 2019.

[9] Menaka.S, Janarthanan, Karpagam,"PROVIDING INTEGRITY VERIFICATION FOR DATA DYNAMICS IN CLOUD," International Journal of Advanced Research in Basic Engineering Sciences and Technology (IJARBEST), ISSN (Online): 2456-5717, Vol. 3, Special Issue 34, 2017.

[10] Udit Satija , Barathram Ramkumar, and M. Sabarimalai Manikandan , "A New Automated Signal Quality-Aware ECG Beat Classification Method for Unsupervised ECG Diagnosis Environments", published by IEEE Sensors Journal,2019,vol19, no1

[11] Naidan Feng, Shaohua Xu , Yongquan Liang, And Kun Liu A "Probabilistic Process Neural Network and Its Application in ECG Classification",published by IEEE access, 2019,vol.7,pp.50432-50439

[12] R. Janarthanan, Doss, S. and S. Baskar. "Optimized unsupervised deep learning assisted reconstructed coder in the on-nodule wearable sensor for human activity recognition", Measurement, Vol. 164, https://doi.org/10.1016/j.measurement.2020.108050, 2020.

[13] Janarthanan, R., Balamurali, R., Annapoorani, A. and Vimala, V., Prediction of rainfall using fuzzy logic. Materials Today: Proceedings, Volume 37, Part 2, 2021, Pages 959-963, 2020.

[14] R Janarthanan, Pachaivannan Partheeban K Somasundaram, P Navin Elamparithi, "A Deep Learning Approach for Prediction of Air Quality Index in a Metropolitan City", Volume 67, 102720, April 2021.

[15] Janarthanan, R., Konar, A. and Chakraborty, A., " Propositional syntax and semantics induced knowledge re-structuring in a fuzzy logic network for ad hoc reasoning," International Journal of Approximate Reasoning, Vol. 82, pp.138-160, 2017. 
[16] Udit Satija , Barathram Ramkumar, and M. Sabarimalai Manikandan "Automated ECG Noise Detection and Classification System for Unsupervised Healthcare Monitoring", published by IEEE Journal Of Biomedical And Health Informatics,2018,vol 22,no3 S.

[17] Zhang, Z. Liu, Y. L. Liu, Y. L. Wang, and X. B. Cui, "Prevalence of stroke and associated risk factors among middle-aged and older farmers in western china. Environmental Health \& Preventive Medicine", 2017,vol. 22, no. 1, pp.6,

[18] I. M. B. Francischetti, A. Cajigas, M. Suhrland, J. M. Farinhas and S. Khader, Incidental primary mediastinal choriocarcinoma diagnosed by endobronchial ultrasound-guided fine needle aspiration in a patient presenting with transient ischemic attack and stroke. Diagnostic Cytopathology, vol. 45, no. 8, pp. 738-743,2017.

[19] M. Anand, N. Balaji, N. Bharathiraja, A. Antonidoss, A controlled framework for reliable multicast routing protocol in mobile ad hoc network, Materials Today: Proceedings,2021,ISSN 2214-7853,https://doi.org/10.1016/j.matpr.2020.10.902.

[20] Sasikumar, B., Naveenraju, D., Anand, K., Hariharan, S., Sudhakaran, P., \& Bharathiraja, N. (2020). Diabetes Prediction Using Sensors By Analysing Skin Temperature. Journal of Engineering Science And Technology, 15(2), 1357-1370. 